These are cynical times and, as Oscar Wilde reminded us, a cynic is someone who knows the price of everything and the value of nothing.

1 Craddock N, Kerr M, Thapar A. What is the core expertise of the psychiatrist? Psychiatrist 2010; 34: 457-60.

2 Cooper B. British psychiatry and its discontents. J R Soc Med 2010; 103 397-402.

3 Salter M, Turner T. Outdoor Psychiatry: A Practical Guide to Community Psychiatry. Elsevier, 2008

Mark Salter is consultant in adult general psychiatry, City and Hackney Centre for Mental Health, London, email: mark.salter@eastlondon.nhs.uk doi: $10.1192 / \mathrm{pb} .35 .2 .74 a$

\section{Improving outcome through patient satisfaction}

Thank you to Dr Whelan and colleagues for their constructive comments ${ }^{1}$ regarding the patient satisfaction scale, PatSat. $^{2}$ The idea for this scale sprung from years of using home-made scales for the yearly appraisal in a flawed attempt to measure the individual doctor's performance in the eyes of the patient. PatSat is therefore uniquely focused on the relationship between the clinician and the individual patient.

As Whelan et al correctly point out, the patient/doctor relationship is only a part of a patient's overall satisfaction with the service, but PatSat provides an evidence-based fundament for the individual clinician to learn about the relative strengths and weaknesses of his or her practice. The idea is that the clinician then can, through supervision, target areas that need further improvement and build on his or her stronger points.

Whelan and colleagues also allude to the importance of treatment outcome and its possible relationship with patient satisfaction. In spite of inherent problems with patient satisfaction questionnaires, such as the 'ceiling effect' (patients often scoring their clinician at the very high end of the spectrum) and poor response rates, the majority of the existing literature on this issue points to a strong correlation between outcome and patient satisfaction, especially with the individual clinician. ${ }^{3}$

The next step would be to investigate the correlation between commonly used, validated rating scales, e.g. the Hamilton Rating Scale for Depression (HRSD) and the Positive and Negative Syndrome Scale (PANSS), and patient satisfaction. In the PatSat scale the clinician has a direct way of testing and re-testing his or her personal impact on patients and the hope is therefore that this will provide an important avenue to improving outcomes for patients.

1 Whelan PJ, Reddy L, Andrews T. Patient satisfaction rating scales vs. Patient-related Outcome and Experience Measures (e-letter). Psychiatrist 2010 (http://pb.rcpsych.org/cgi/eletters/34/11/485).

2 Hansen LK, Vincent S, Harris S, David E, Surafudheen S, Kingdon D. A patient satisfaction rating scale for psychiatric service users. Psychiatrist 2010; 34: 485-8.

3 Day JC, Bentall RP, Roberts C, Randall F, Rogers A, Cattell D, et al. Attitudes toward antipsychotic medication: the impact of clinical variables and relationships with health professionals. Arch Gen Psychiatry 2005; 62: 717-24.

Lars K. Hansen is consultant psychiatrist, Fairway's House, Hampshire Partnership NHS Foundation Trust, email: Ih4@soton.ac.uk, David Kingdon is Professor of Psychiatry, University of Southampton, Hampshire Partnership NHS Foundation Trust

doi: $10.1192 / \mathrm{pb} .35 .2 .75$

\section{What makes a good psychiatrist?}

We welcome the restatement by Craddock et al of the depth and diversity of 'added value' that the psychiatrist brings to mental health services.

In 2007, we undertook a standardised survey of the views of psychiatrists, mental health nurses and patients on what were the key attributes a psychiatrist should possess, which we entitled 'What makes a good psychiatrist?'

Overall, 244 psychiatrists, 70 nurses and 86 out-patients from across Scotland completed the survey. The top four key attributes to being 'a good psychiatrist' identified by the survey were different for the three groups.

Psychiatrists ranked clinical knowledge as the most important attribute (47.5\%); 'communicates clearly' came second (20\%), 'interested in people' third (19\%) and 'honest and trustworthy' fourth (18\%).

Top four attributes identified by nurses were: approachable (29\%), clinical knowledge (27\%), communicates clearly (24\%) and good listener (14\%).

For patients, the ranking was different still: good listener (41\%), approachable (25\%), treats patients as equals (23\%) and non-judgemental (16\%).

There are echoes of various guideline documents in these results (e.g. New Ways of Working, Good Medical Practice) and of a similar survey from Ireland. ${ }^{2}$ Clearly, communication skills and individual values and attitudes are important, as is clinical knowledge. We believe that the patients did not rate clinical knowledge highly as they simply assume it to be there, even if the depth of general medical knowledge is not always appreciated. All three groups questioned did not feel that interests outside of psychiatry, or being well presented, were important professional attributes.

1 Craddock N, Kerr M, Thapar A. What is the core expertise of the psychiatrist? Psychiatrist 2010; 34: 457-60.

2 Cullen W, Bury G, Leahy M. What makes a good doctor? A crosssectional survey of public opinion. Ir Med J 2003; 96(2): 38-41.

Mark Taylor is a consultant psychiatrist, Intensive Home Treatment Team, Ballenden House, Edinburgh, email: marktaylor2@nhs.net, Alison MacRae is a consultant psychiatrist, NHS Ayrshire and Arran, Scotland

doi: $10.1192 / p b .35 .2 .75 a$

\section{Are crisis resolution teams toxic?}

I read with interest the paper by Forbes et al, which investigated the impact of a crisis resolution service. I am intrigued by their finding that the introduction of the crisis service was followed by an unexpected increase in the absolute numbers of patients detained under the Mental Health Act. In their discussion a number of possible explanations are explored. However, I believe there is one possible explanation, which is not fully discussed, although it is perhaps hinted at in the clinical implications section of their abstract. This is that the intervention might have a negative impact on some patients.

This is now the third study to find this association, ${ }^{2,3}$ with only one group failing to replicate it. ${ }^{4}$ Tyrer et $a l^{3}$ explicitly and at some length discuss the notion that negative effects on some patients of this type of service are one of the most plausible explanations for the increase in compulsory admissions. Furthermore, they suggest that any benefit from crisis 